\title{
Molecular Profile of Integrase gene intI and Carbapenem gene in Aeromonas sobria Isolates
}

\author{
Hawraa Natiq Kabroot AL-Fatlawy ${ }^{1}$, Taghreed Abdul Al-Kareem Al-Makhzoomy ${ }^{2}$, \\ Mohammed Abdulaali Al-Hakeem ${ }^{3}$
}

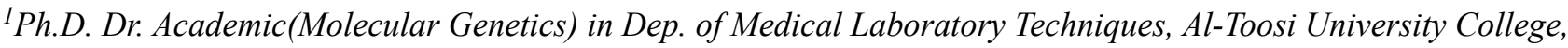
Najaf, IRAQ, ${ }^{2}$ M.Sc.(Microbiology) in Laboratory Investigation department, Faculty of Science, University of Kufa, Najaf, IRAQ. ${ }^{3}$ B.Sc. in Biology department, Faculty of Science, University of Kufa, Najaf, IRAQ

\begin{abstract}
The current study includes the detection of Aeromonas sobria isolates were obtained from total 157 samples involved the clinical samples (diarrhea samples) from patients suffering from diarrheal infections during the period from April 2017 to November 2017. at AL-Central Health Laboratory in Najaf governorate. These isolates were diagnosed by four method as (Culture method, biochemical tests, Vitek@2GN cards system and Polymerase chain reaction (PCR) technique).TheVitek@2GN cards system was the best method for diagnosis, which have led to isolate and diagnosis of 33 isolate. Multidrug resistance (MDR) of bacteria were detected by MIC testing and performed with the automated VITEK@2 GN/AST compact system. A clear variation was observed in their susceptibility to 13 antibiotics disks, these isolates of A.sobria were revealed resistance for some antibiotics such as Penicillin class Methicillin, Amoxicillin, Ampicillin, Penicillin (100\%), and (91.6\%) for Carbenicillin, and high rates of resistance (100\%) to Cephalosporins that represented by Ceftazidime, Cefotaxime, Cefoxitin and Cefepime. The investigation of mobile genetic elements among isolates demonstrated thatA.sobria have been Class I integron genes (class 1 integron represented by integrase intI1, and IMP genes).
\end{abstract}

Keywords: Aeromonas soria, IMP gene, integrase intI1, Antibiotics and imipenem.

\section{Introduction}

Aeromonas sobria are species of the genus Aeromonas, which belongs to the family Aeromonadaceae that received increasing attention opportunistic pathogens because of its association with both diarrheal and extra intestinal infection in human disease especially in children and persons with impaired immune system ${ }^{1,2,3}$. Multidrug resistance (MDR) was reported in the genus of Aeromonas ${ }^{4}$. A.hydrophilaand A.sobriaare anantibiotic resistant a variety of antibiotics have been used to treat infection caused by A.hydrophila

\section{Corresponding Author:}

\section{Hawraa Natiq Kabroot AL-Fatlawy}

Ph.D. Dr. Academic(Molecular Genetics) in Dep. of

Medical Laboratory Techniques, Al-Toosi University

College, Najaf, IRAQ

e-mail: dr.hawraank9@altoosi.edu.iq and A.sobria. The high occurrence of (MDR) observed in Aeromonas spp. ${ }^{5,6}$. The antibiotic resistance genes that integrons capture are located on gene cassettes. Aeromonas spp. have been contain Class I integrin ${ }^{7}$. Integrons may be found as part of mobile genetic elements such as plasmids and transposons. Integrons can also be found in chromosomes. The aim of studyfor this purpose, the steps were: 1- Isolation and identification of A.sobria in clinical and in Najaf by VITEK@2 GN/ID card system. 2- Detection the Antimicrobial susceptibility of A.hydrophila and A.sobria isolates using antibiotics disks and MIC/AST. 3- Characterization of Class I integron and related mobile genetic elements (MGE) among MDR isolates. And determination of some MDR and $\beta$-lactamase resistance genes such as $\left(b l a_{\mathrm{IMP}}\right)$.

\section{Materials and Method}

- Identification of Aeromona: Phenotypic properties were recorded on microscopic characteristics by 
Gram's stain was used to examine the isolated bacteria for studying the microscopic characteristics such as gram reaction, shape, motile and the media that are used (TCBS agar; MacConkey agar and blood agar) for primary identification of Aeromona. Also Biochemical tests used Catalase test (3\% Hydrogen Peroxide), Oxidase test, Indole Production test, Simmon's Citrate test and Motility test were all these tests and urease test result according to studies $^{8,9}$.

- Identification of Aeromonas by VITEK@2 GNID System: The identified Aeromonas ssp isolates were confirmed with the automated VITEK@2 compact system by using GN/ID cards. The GN ID card is based on established biochemical method (64 reaction) and newly developed substrates, measuring various metabolic activities.

- Antibiotics susceptibility: Minimum Inhibitory Concentration (MIC) antibiotics were used by VITEK@2/AST, such as (AST-GN084, ASTGN093 Card system), as mentioned in antibiotics disks were used 13 antibiotic of many class and sub-class antibiotics (Penicillins, Cephems, Monobactams, Nitrofurans, Quinolones, Ansamycin, $\quad \beta$-lactamase, $\quad$ Carbapenem, Tetracycline, Aminoglycosides, Phenicols, Folate pathway inhibitors and Lipopeptide).

\section{Results And Discussion}

\section{Isolation and Identification Culture and}

Biochemical Tests: The isolation and identification of Aeromonas sobria showed that only 33 isolate were positive based on the morphological characteristics of the colonies on TCBS, MacConkey agar, Aeromonas media and blood agar media These isolates were smooth yellow, shiny, flat, about $2-3 \mathrm{~mm}$ in diameter colonies on TCBS (Fig. 1), while they were small and pale colonies on MacConkey's agar when incubated for $24 \mathrm{~h}$. While microscopic examination of cultures showed that the bacteria were gram-negative slightly curved rods, non-spore forming cells, arranged as single or double of bacterium bacilli. The classification of Aeromonas has been confusing because of lack of matching between phenotypic and genotypic characteristics of species and multiple method that are required for accurate taxonomy ${ }^{10}$.

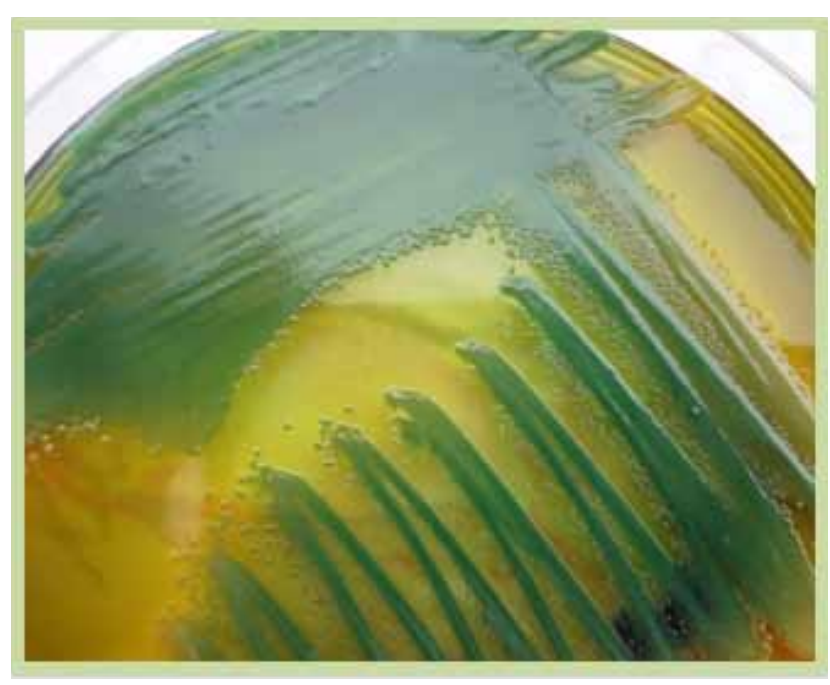

Fig. 1: A.sobria isolate on Aeromonas isolation agar and

On the other hand, the results of biochemical tests referred to that not all were positive to oxidase and catalase tests. The positive isolates were characterized with the ability to ferment the glucose only on KIA, so the isolates gave alkaline slant with acid bottom without $\mathrm{H} 2 \mathrm{~S}$ or $\mathrm{CO}^{2}$ production. Also, isolates showed positive results to simmon's citrate and negative to urease test [Table 1]. According to these biochemical tests only 33 stool samples showed positive result as A.sobria. This result was predicted by previous studies ${ }^{6,11,12}$.

Table 1: Biochemical tests for Aeromonas

\begin{tabular}{|c|l|c|}
\hline No. & Tests & A.sobria \\
\hline 1 & Oxidase test & + \\
\hline 2 & Kligler iron agar & Alk/Acid with gas \\
\hline 3 & Indole test & + \\
\hline 4 & Citrate test & + \\
\hline 5 & Methyl red test. & + \\
\hline 6 & VP & + \\
\hline
\end{tabular}

The identification by VITEK@2 was performed with the automated VITEK@2 system using GN-ID cards which contained 64 biochemical tests. The results were demonstrated 33 A.sobria isolates were confirmed with ID message confidence level ranging excellent (Probability percentage from 94 to $99.7 \%$ ). 


\section{Molecular Identification by PCR Technique:}

\section{Antimicrobial Susceptibility Determination:}

\section{A. Minimum Inhibitory Concentration of} Aeromonas: MIC testing was performed with the automatedVITEK@2 GN/AST compact system.The results of the study revealed that allA.sobriaisolates were resistant to minimum of many classes of antibiotics (MIC) to which they are tested. Hence, all isolates were considered to be multidrug resistant (MDR), revealed that the resistant of $A$.sobria isolates to Penicillins (Ampicillin, Amoxicillin/Clavulanic, Piperacillin and Piperacillin-Tazobactam) were recorded in $100 \%$ for all isolates. The resistance to $\beta$-lactam $/ \beta$-lactamase inhibitor combinations was appeared in $100 \%$ of Aeromonas isolates. MDR of A.sobria isolates were represented by resistance to twelve class and sub class of antibiotic. All isolates appeared resistance to (Penicillin class) Methicillin, Amoxicillin, Ampicillin, Penicillin $(100 \%)$, and $(91.6 \%)$ for Carbenicillin. The study also revealed a high rates of resistance to Cephalosporins that represented by Ceftazidime, Cefotaxime, Cefoxitin and Cefepime were detected in $(100 \%)$. Resistance to all $\beta$-lactam/ $\beta$-lactamase inhibitor combinations includingAmoxicillinClavulanic acid and Pipracillin-Tazobactam $(100 \%)$. The results of resistance isolates appeared high resistance to Imipenem (75\%), Meropenem (77\%) and Ertapenem (80\%) of Carbapenem class, were effective against the majority of these isolates, these results agreed with other studies such as ${ }^{4,13}$. The human populations within these regions are at risk of exposure to antimicrobial resistant bacteria, and thereby disseminating antimicrobial resistance (ARGs) genes ${ }^{14}$.

\section{Molecular Detection of Resistance and Integron} Class I Genes: All A.sobria isolates were detected for the present of ESBL genes. The results revealed that isolates yielded amplification products with specific primers for types of extended-spectrum- $\beta$-lactamase(ESBL) and metallo-beta-lactamase (Carbapenem) antibiotics genes. All isolates in the present study were tested phenotypically of ESBL and Carbapenem production by MIC method and genotype. However, gene in the families only IMP were examined in the present study. Detection of these genes was performed by PCR technique. The results revealed that out of the 29 A.sobriaisolates contained

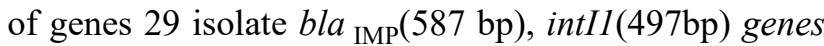
as mentioned in [fig. 2] and [fig. 3]. Previous studies unquestionably established the role ( $>85 \%$ of isolates) of many Aeromonas spp. such as A. hydrophila, A. Sobria, A.caviaeand $A$. veroniiin diarrhea ${ }^{4,15}$. Deng et $a l^{16}$ confirmed that Aeromonas strains containing multiple drug-resistance integrons, and these data suggests that surveillance for antimicrobial resistance of animal origin and responsible use of antimicrobials in aquaculture is necessary in these farms. Current results are agreed of Aeromonas produced class I integronsgenes with other studies ${ }^{7,17,18}$.

\section{0,000}

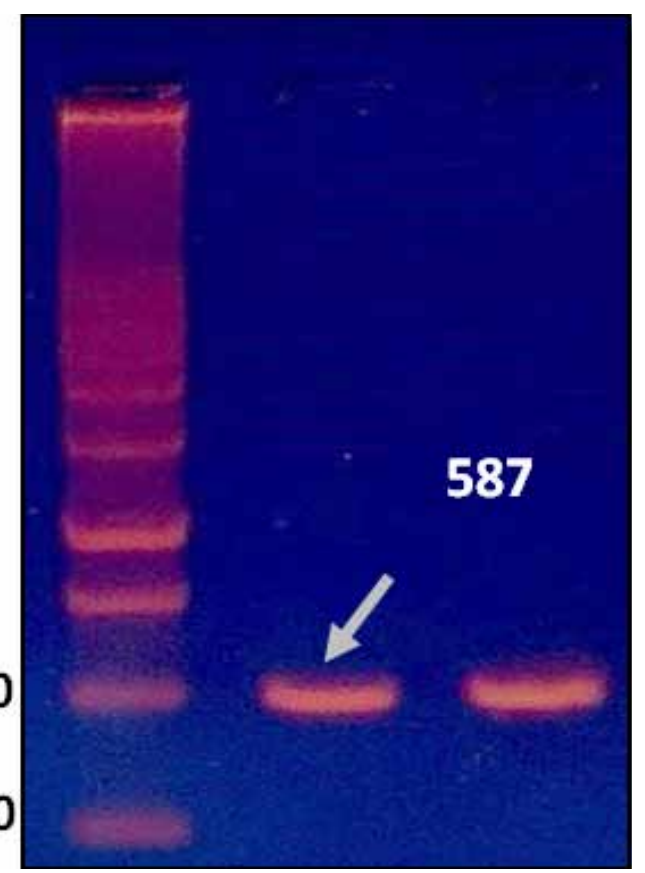

Fig. 3: Agarose Gel Electrophoresis (1.5\%) of PCR products of intI1(497 bp) gene of .sobria isolates for (45) $\mathrm{min}$ at (100) volt. 


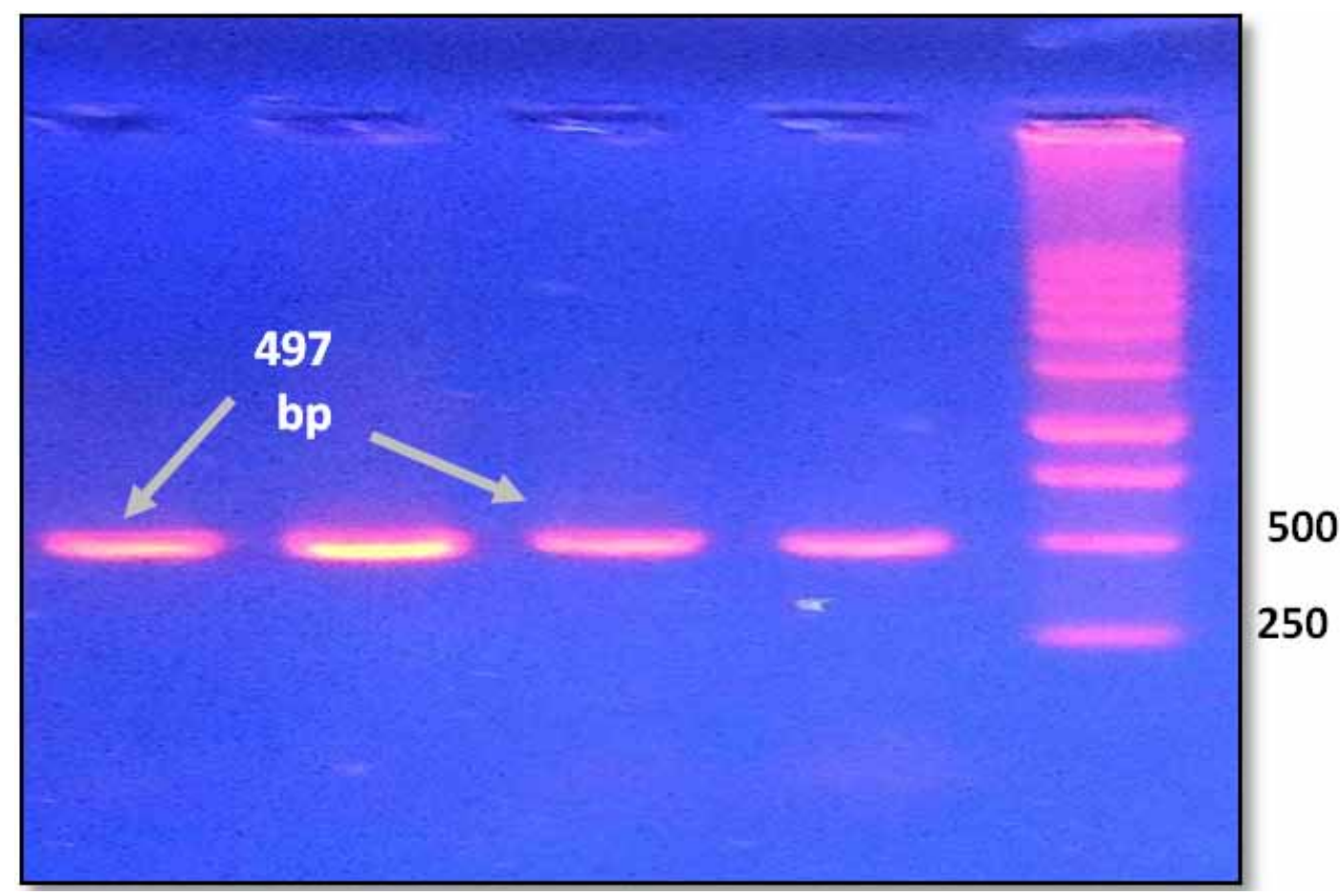

Fig. 2: Agarose Gel Electrophoresis (1.5\%) of PCR products of IMP Genes (587bp) of $A . s o b r i a$ isolates for (45) min at (100) volt.

\section{Conclusion}

The frequency of A.sobria isolates in Najaf were higher among local clinical isolates. Identification by VITEK@2GN card system and Molecular techniques are necessary for detection of pathogenic bacteria among clinical isolates. Molecular characterization of Class I integrongenes, genetic elements (MGE) among MDR isolates were found in most Aeromonas isolates. A.sobriawas multidrug resistance by shown a great emergence of MDR isolates among strains isolated and resistance to twelve class and sub class of antibiotic Methicillin, Amoxicillin, Ampicillin, Penicillin, Ceftazidime, Cefotaxime, Cefoxitin and Cefepime, Amoxicillin Clavulanic acid and PipracillinTazobactam, Rifampin.

Acknowledgement: We would like to thank all laboratories staffs at dep. Of Medical Laboratory Techniques of Al-Toosi University College, and laboratories staffs at Faculty of Science, University of Kufa, Najaf, Iraq.

\section{Funding: None}

Conflict of Interest: All authors are no conflict of interest.
Contribution: The authors in this work have approved it for publication in your journal .

Data: All data were analyzed during this work are included in the manuscript.

Ethics Statement: The work does not contain any study with animals performed or human participant by any of the author.

\section{References}

1. Naharro, G.; Riano, J.; de Castro, L.; Alvarez, S.; Luengo, J.M. Aeromonas: Molecular Detection of Foodborne Pathogens (Liu D. Ed.). CRC Press. North Ryde, 2009; 273-289.

2. Uche, O.A. and Johnkennedy, N. Prevalence of Aeromonas species among patients attending general hospital Owerri. J.AMS, 2014; 1(4).1:10.

3. Al-Fatlawy, H.N.K and AL-Hadrawy, H.A. Isolation and Characterization of A. hydrophila from the Al-Jadryia River in Baghdad (Iraq). American Journal of Educational Research, 2014, Vol. 2, No. 8, 658-662. DOI:10.12691/education-2-8-14.

4. Bakhtiar-Lijon, Md;Khatun, M.;Islam, A.;Khatun, M. and Islam, A.Detection of multidrug resistance 
Aeromonas hydrophilain farm raised fresh water prawns.J. Adv. Vet. Anim. Res., 2016; 2(4): 469474.

5. Ndi, O.L., Barton, M.D. Incidence of class 1 integron and other antibiotic resistance determinants in Aeromonas spp. from rainbow trout farms in Australia. J. Fish Dis. 2011; 34, 589-599.

6. AL-Fatlawy, H.N.K; Aldahhan, H.A and Alsaadi, A.H. Phylogenetic of ERIC-DNA Fingerprinting and New Sequencing of Aeromonas Species and V. Cholerae DNA. American Journal of Applied Sciences 2017, 14 (10): 955.964. DOI: 10.3844/ ajassp.2017.955.964.

7. Mazel, D. Integrons: agents of bacterial evolution. Nat Rev Microbiol; 2006; 4:608-620.

8. Jawetz, E., Melnick J.I. and AdelbergE.A.. Medical Microbiology, 2016; 27th Edn., Appleton and Lange U.S.A.

9. WHO. World Health Organization, Microbiological agents in drinking water.2000Vibrio cholerae ( $2^{\text {nd }}$ ed.).

10. Králová, S.; Staňková, E.; and Sedláček, I. Classification of Aeromonas spp. isolated from water and clinical sources and distribution of virulence genes. Folia Microbiol, 2016; 61(6):513521 . DOI 10.1007/s12223-016-0464-9.

11. AL-Fatlawy, H.N.Kand Al-Ammar, M.H. Study of Some Virulence Factors of Aeromonas Hydrophila Isolated from Clinical Samples (Iraq). International Journal of Science and Engineering Investigations, Volume 2, Issue 21, October 2013. Paper ID: 22113-16.

12. Yang Q-H, Zhou C, Lin Q, Lu Z, He L-B, Guo S-L. (2017). Draft genome sequence of
Aeromonas sobriastrain 08005, isolated from sick Rana catesbeiana. Genome Announc, 2017; 5(2): e01352-16.

13. Igbinosa, I. H.; Igbinosa, E.O. and Okoh, A.I. "Virulence gene markers and biofilm formation of Aeromonas species recovered from cow faeces."Journal of Clinical and Cell Immunology, 2014; 5(5) 184.

14. Usui, M.;Tagaki, C.;Fukuda, A.;Okubo, T.;Boonla, C.;Suzuki, S.;Seki, K.; Takada, H.; and Yutaka Tamura, Y. Use of Aeromonas spp. as General Indicators of Antimicrobial Susceptibility among Bacteria in Aquatic Environments in Thailand. Frontiers in Microbiology, 2016; 7 | Article 710 7:710. doi: 10.3389/fmicb.2016.00710.

15. Janda, J.M. and Sharon, L. The genus Aeromonas: taxonomy, pathogenicity, and infection. Clin Microbiol Rev, 2010;23:35-73.

16. Deng, Y.; Bao, X.; Ji, L.; Chen, L.; Liu, J.; Miao, J.; Chen, D.; Bian, H.; Li, Y. and Yu, G. Resistance integrons: class 1, 2 and 3Integrons. Ann Clin Microbiol Antimicrob(2015) 14:45.

17. Malek,M.M., Amer,F.A.,Allam,A.A.,El-Sokkary, R. H., Gheith, T., and Arafa, M. A. Occurrence of classes Iand II integrons in Enterobacteriaceae collected from ZagazigUniversity Hospitals, Egypt. Front. Microbiol, 2015; 6:601. doi: 10.3389/ fmicb.2015.00601.

18. Al-Hadrawi, H.; Al-Harmoosh R.; Al-Fatlawy, H.N.K. Isolation and Identification of Streptococcus pneumonia Causing Respiratory and Thoracic Diseases in Najaf Province.J Pure Appl Microbiol, 2019; 13(4).doi 10.22207/JPAM.13.4. 